\title{
Nonlinear Analysis of GMSK Carrier Phase Recovery Loop
}

\author{
Mohamed Amine Jhaidri* ${ }^{\dagger}$, Christophe Laot ${ }^{*}$, Alain Thomas ${ }^{\dagger}$ \\ *Institut Mines-Telecom, Telecom Bretagne, Lab-STICC, UMR CNRS 6285, 29238 Brest, France \\ ${ }^{\dagger}$ Zodiac Data Systems, 5 Avenue des Andes, 91940 Les Ulis, France
}

\begin{abstract}
Deep space communication systems operate in the presence of high atmospheric attenuation and the capacity of the on-board energy generator is limited which produces a very low signal to noise ratio (SNR) at the reception. In order to satisfy the required transmission quality of deep space mission in terms of power and spectral efficiency, a coherent demodulation is mandatory. Coherent demodulation performance depends on the carrier phase synchronizer ability and its robustness against transmission constraints such as time-varying Doppler effect (Doppler rate). An efficient and well designed carrier phase recovery scheme is required to assure the synchronization. This paper analyses the performance of a blind carrier phase synchronizer derived from the maximum a posteriori criterion (MAP) and Laurent expansion for precoded Gaussian Minimum Shift Keying (GMSK) modulation. Due to the scarcity of spectral resources allocated for deep space transmission, the data-aided approach is not allowed. In the previous works, the carrier phase recovery system performance was evaluated in tracking phase without assessing its capacity in locking phase. In this paper, we present a more complete analysis of a GMSK carrier phase synchronizer covering the nonlinear acquisition phase and the theoretical operating limits in terms of SNR and input phase offset (Doppler, Doppler rate) in a closed-loop structure.
\end{abstract}

Index Terms-Carrier phase synchronization, deep space communication, GMSK modulation, nonlinear acquisition, phase detector S-curve, phase plane analysis.

\section{INTRODUCTION}

Spectrum resources allocated for deep space missions are limited (X-band at $8 \mathrm{GHz}$ ). In order to optimize the spectral efficiency, the Consultative Committee for Space Data System (CCSDS) recommends [1] precoded GMSK modulation with a Gaussian filter 3-dB bandwidth-bit period product $B T_{b}=0.5$ for Category B missions (deep space missions) and GMSK $B T_{b}=0.25$ for Category A missions (low altitude missions). GMSK is a continuous phase modulation (CPM) having several interesting properties such as constant envelope which allows the amplifier to operate in full-saturation mode and maximize conversion efficiency without undergoing any form of spectral distortion. GMSK $B T_{b}=0.5$ outperforms binary phase shift keying (BPSK) in terms of bandwidth efficiency with a bit error rate performance nearly identical in the case of a perfect carrier phase synchronization. Synchronization is an essential task in any coherent digital communication system. In most cases, this task is provided by a digital phaselocked loop (DPLL). The DPLL is a good trade-off between implementation complexity and performance. By definition,
DPLL is a closed-loop carrier phase estimator including three basic elements:

1) Phase detector (PD)

2) Loop filter (LF)

3) Numerically controlled oscillator (NCO)

In order to define the PLL model and correctly set the system parameters (equivalent noise bandwidth, damping factor...), which is the main objective of this paper, we need to characterize the phase detector by the so-called S-curve. By definition, the S-curve $S(\theta)$ is the averaged value of the phase error detector output (in open-loop structure) in the presence of a constant phase offset at its input. In the tracking phase (near zero phase error), the phase error detector is represented by $\alpha$ the slope at the origin of the $\mathrm{S}$-curve. In the acquisition phase, this approximation is no longer valid and the phase error detector must be represented by the entire S-curve analytic expression in the nonlinear carrier phase loop model.

Using Laurent expansion [2] of GMSK modulated signal, several carrier phase detectors were proposed in previous works and their tracking performance was evaluated in terms of squaring loss and bit error rate. In [3], the author derives the optimal blind carrier phase detector by applying the maximum a posteriori phase estimation method. In order to reduce the complexity of MAP carrier phase detector of [3], the authors in [4] propose two simplified versions based on high $(\tanh (x) \simeq$ $\operatorname{sign}(x))$ and low $(\tanh (x) \simeq x)$ SNR approximations of the nonlinear hyperbolic tangent function. In [5], the simplified GMSK phase detector with the low SNR approximation has been compared in terms of detection threshold and BER performance to a modified offset quadrature phase shift keying (OQPSK) phase detector adapted to GMSK signal.

Taking into account the deep space data transmission constraints (very low SNR), we consider in this work the GMSK phase detector with the low SNR tanh function approximation of [4]. The contributions of our paper are the following: first, we derive the analytic S-curve expression of this detector. To the best of our knowledge, this expression does not exist in the literature. Second, using the derived analytical expression of the S-curve, we present a new study of the nonlinear behavior of a phase locked loop with a phase error detector having the following form: $S(\theta) \propto \sin (2 \theta)$. This study is based on the phase plane method [6] which is a graphical method for nonlinear system analysis. In the literature [6] we find several 
analyses of the phase locked loop (PLL) nonlinear behavior with sinusoidal phase error detector $S(\theta) \propto \sin (\theta)$ which is not the case of the phase error detector considered in this work.

The paper is organized as follows: section II describes the GSMK data transmission system and Laurent expansion. We derive in section III the phase error expression using the MAP criterion and the low SNR approximation of the tanh function. In section IV, we detail a mathematical determination of the S-curve expression of the considered detector. Section $\mathrm{V}$ is dedicated to the nonlinear analysis and the last section concludes the paper.

\section{SySTEM DESCRIPTION}

The deep space transmission channel is a mono-path channel disturbed by an additive white Gaussian noise (AWGN). Note that in this work we are interested in the carrier phase synchronization and the clock offset is assumed to be perfectly estimated and equal to zero. Fig.1 shows a block diagram of the precoded GMSK communication system. The system consists of a binary data source (delivering the information bits $d_{k}$ ), a data precoder, a GMSK modulator and a coherent receiver with carrier phase recovery scheme.

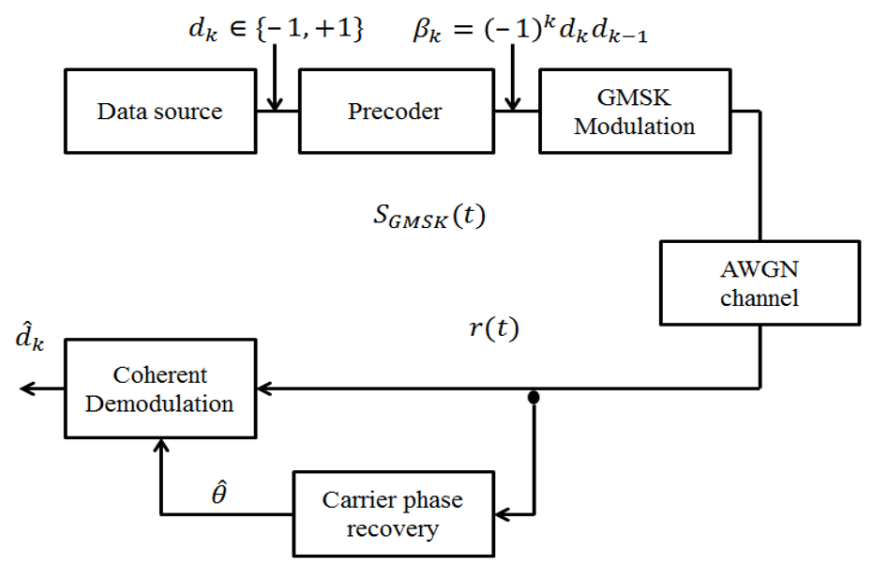

Figure 1. GMSK communication system

GMSK modulation $\left(B T_{b}=0.5\right)$ is a partial-response CPM scheme obtained by filtering the input binary NRZ data symbols $d_{k}$ with a filter having a gaussian impulse response and frequency modulating the filter output onto a carrier signal with a modulation index $h=0.5 . B$ is the $3-\mathrm{dB}$ bandwidth of the gaussian filter and $T_{b}$ is the bit period. Using the Laurent expansion for binary CPM [2] modulation, the complex envelope of the GMSK $B T_{b}=0.5$ signal can be exactly represented by a superposition of two amplitude-modulation pulses (AMP) (1). For this $B T_{b}$ value, the Gaussian filter is truncated in time to two bit intervals.

$S_{G M S K}(t)=\sqrt{\frac{E_{b}}{T_{b}}} \sum_{k=0}^{\infty}\left\{a_{0, k} C_{0}\left(t-k T_{b}\right)+a_{1, k} C_{1}\left(t-k T_{b}\right)\right\}$

where $E_{b}$ is average energy per bit, $\left\{a_{0, k}, a_{1, k}\right\}$ are the data symbols and $\left\{C_{0}, C_{1}\right\}$ are the Laurent pulses. the duration of the main pulse $C_{0}(t)$ is $3 T_{b}$ while the second pulse $C_{1}(t)$ has a duration of $T_{b}$ and:

$$
\begin{gathered}
a_{0, k}=\prod_{n=0}^{k} j \beta_{n}=j \beta_{k} a_{0, k-1}, \\
a_{1, k}=j \beta_{k} a_{0, k-2}
\end{gathered}
$$

and

$$
a_{0,-1}=1, a_{0,-2}=j
$$

with $\beta_{k}=(-1)^{k} d_{k} d_{k-1}$, obtained by differential encoding of the information data bits $d_{k}$ as presented in Fig. 1.

\section{CARRIER PHASE ESTIMATION}

The received signal $r(t)$ is described by:

$$
r(t)=S_{G M S K}(t) e^{j \theta(t)}+n(t)
$$

where $n(t)$ is an additive white Gaussian noise (AWGN) with single-side PSD $N_{0} \mathrm{~W} / \mathrm{Hz}$ and $S_{G M S K}(t)$ is given by (1). The phase difference $\phi(t)$ between the carrier phase $\theta(t)$ and the phase generated by the receiver local NCO $\hat{\theta}(t)$ degrades the system BER performance. Therefore, to satisfy the required transmission performance, we need to estimate the carrier phase at the reception and use it to coherently demodulate the received signal and eliminate the BER loss. According to the MAP estimation criterion, the most likely phase $\hat{\theta}(t)$ is that which maximizes the a posteriori probability $p(\hat{\theta}(t) \mid r(t))$. Moreover, it is assumed that $\theta(t)$ is uniformly distributed over the interval $[-\pi, \pi]$, which means that maximizing $p(\hat{\theta}(t) \mid r(t))$ is equivalent to maximizing $p(r(t) \mid \hat{\theta}(t))$ (maximum-likelihood ML estimation). In the case of GMSK $B T_{b}=0.5,99.97 \%$ of the signal energy is carried by the first Laurent pulse $C_{0}$. Therefore, in order to simplify the error phase expression, we ignore the second Laurent pulse $C_{1}$. For an AWGN channel and a constant envelope modulation the likelihood probability has the form:

$$
p(r(t) \mid \hat{\theta}(t), \hat{\mathbf{a}})=C \exp \left(\frac{2}{N_{0}} \Re\left(\int_{0}^{T_{0}} r(t) s^{*}(t, \hat{\theta}, \hat{\mathbf{a}}) d t\right)\right.
$$

where

$$
\left.s(t, \hat{\theta}, \hat{\mathbf{a}})=\sqrt{\frac{E_{b}}{T_{b}}} \sum_{k=0}^{\infty}\left\{\hat{a}_{0, k} C_{0}\left(t-k T_{b}\right)\right)\right\} e^{j \hat{\theta}(t)}
$$

where $\left[0, T_{0}\right]$ is the observation interval, $\{\hat{\mathbf{a}}, \hat{\theta}(t)\}$ are the estimates of the parameters that need to be determined and $C$ is a normalization constant. 


\section{A. Closed-loop carrier phase estimation}

To arrive at the blind closed loop synchronizer, we consider a sliding window implementation of the MAP carrier estimator. In other words, we decompose the observation period $\left[0, T_{0}\right]$ into a set of sub-periods with a time length equal to $k_{b} T_{b}$. During the $k^{t h}$ sub-observation period $\left[k T_{b},\left(k+K_{b}\right) T_{b}\right]$ we assume that the carrier phase $\theta(t)$ is constant and equal to $\theta_{k}\left(\theta(t)\right.$ can be a time-varying phase over the interval $\left.\left[0, T_{0}\right]\right)$. Afterward, at each instant $k T_{b}$ we take the natural logarithm of the likelihood probability (6) calculated over the time interval $k T_{b} \leqslant t \leqslant\left(k+K_{b}\right) T_{b}$ and averaged over the data sequence $\hat{a}$, differentiate it with respect to the estimated phase at this interval $\hat{\theta}_{k}$ and finally use this as an estimation error signal $e\left(\phi_{k}\right)$, where $\phi_{k}=\theta_{k}-\hat{\theta}_{k}$ : is the phase offset. By tacking $K_{b}=4$ and considering only the even instants ( $k$ is even), we obtain:

$$
e_{k}\left(\phi_{k}\right)=-\Re\left(I_{k}\right) \tanh \left(\Im\left(I_{k}\right)\right)+\Im\left(I_{k+1}\right) \tanh \left(\Re\left(I_{k+1}\right)\right)
$$

where

$$
I_{k}=\frac{2}{N_{0}} \sqrt{\frac{E_{b}}{T_{b}}}\left\{\int r(t) C_{0}\left(t-k T_{b}\right) d t\right\} e^{-j \hat{\theta}_{k}}
$$

which is the matched filter output sampled at $k T_{b}$ and multiplied by $\left(2 / N_{0}\right) \sqrt{E_{b} / T_{b}}$ and the estimated phase. At very low signal to noise ratio, the nonlinear hyperbolic tangent function can be approximated by a linear function as $\tanh (x) \simeq x$. Once the hyperbolic tangent function is approximated by the linear function, the multiplication by $2 / N_{0}$, which was used to place the matched filter output in the appropriate region of the tanh function (linear or constant region), becomes useless. Therefore, we introduced the new variables $I^{\prime}=\left(N_{0} / 2\right) I$ (which is the sampled matched filter output multiplied only by the signal power and the estimated phase) and the approximated phase error signal becomes:

$$
e_{k}^{\prime}\left(\phi_{k}\right)=-\Re\left(I_{k}^{\prime}\right) \Im\left(I_{k}^{\prime}\right)+\Im\left(I_{k+1}^{\prime}\right) \Re\left(I_{k+1}^{\prime}\right)
$$

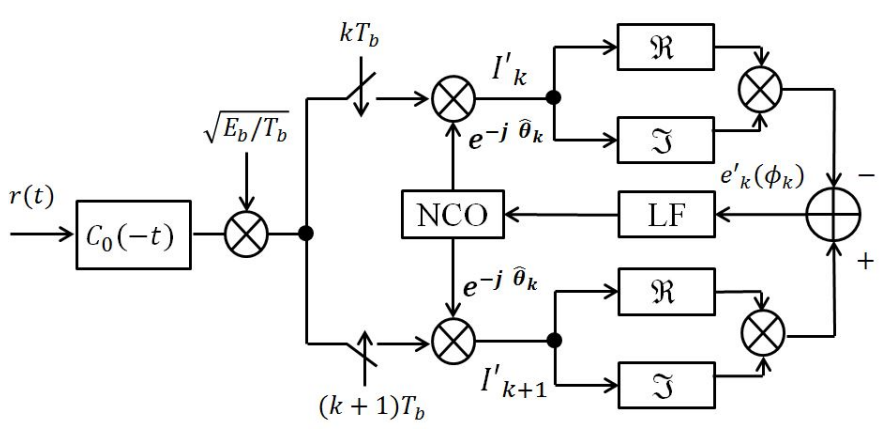

Figure 2. Carrier phase recovery scheme for GMSK $B T_{b}=0.5$

Fig.(2) illustrates a block diagram for the closed-loop carrier phase estimator. The system consists of a phase error detector (10), a first order proportional-integrator loop filter whose transfer function $F(s)$ is described by (11) and a NCO.

$$
F(s)=K_{f}\left(1+\frac{a}{s}\right)
$$

where $K_{f}$ is the loop filter gain and $a$ is the integrator gain. These parameters will be set according to the mission constraints (Doppler, Doppler rate, phase error variance, acquisition time). The main objective of the nonlinear analysis, which will be detailed in the last section, is to express the operating limits of the carrier phase recovery scheme as a function of the loop parameters.

\section{Derivation of the S-Curve}

To define the equivalent model of the carrier phase recovery loop and investigate its performance using system model analysis, we need to characterize the phase detector by the so-called S-curve. By definition (12) the S-cure is the average value of the phase detector output in the presence of a constant phase offset $\phi$ between the carrier signal and the receiver N.C.O in an open loop structure.

$$
S(\phi)=E\left[e_{k}^{\prime}(\phi) \mid \phi\right]
$$

It can be shown that

$$
E\left[e_{k}^{\prime}(\phi) \mid \phi\right]=2 E\left[-\Re\left(I_{k}^{\prime}\right) \Im\left(I_{k}^{\prime}\right)\right]=2 E\left[\Re\left(I_{k+1}^{\prime}\right) \Im\left(I_{k+1}^{\prime}\right)\right]
$$

By Substituting (5) in (9) and multiplying the result by $N_{0} / 2$, we obtain

$\Re\left(I_{k}^{\prime}\right)=E_{b}\left(\left(d_{k-1}+d_{k+1}\right) R(1) \cos (\phi)-d_{k} R(0) \sin (\phi)\right)+n_{k, I}$

$\Im\left(I_{k}^{\prime}\right)=E_{b}\left(\left(d_{k-1}+d_{k+1}\right) R(1) \sin (\phi)+d_{k} R(0) \cos (\phi)\right)+n_{k, Q}$

where

$$
R(l)=\frac{1}{T_{b}} \int_{0}^{3 T_{b}} C_{0}(t) C_{0}(t-l T b) d t
$$

$n_{k, I}$ and $n_{k, Q}$ are uncorrelated zero mean Gaussian random variables with variance $\sigma_{N}^{2}=R(0) E_{b} N_{0} / 2$. Multiplying $\Re\left(I_{k}^{\prime}\right)$ and $\Im\left(I_{k}^{\prime}\right)$ and substituting the result into (13) produce the phase detector S-curve:

$$
S(\phi)=E_{b}^{2}\left(R^{2}(0)-2 R^{2}(1)\right) \sin (2 \phi)
$$

The slope at the origin of the S-curve is given by

$$
\alpha=\left.\frac{d S(\phi)}{d \phi)}\right|_{\phi=0}=2 E_{b}^{2}\left(R^{2}(0)-2 R^{2}(1)\right)
$$

The S-curve of the detector is plotted versus the phase error in Fig.3. Notice that the S-curve $S(\phi)$ is $\pi$-periodic and the synchronizer can lock either on the correct phase $(\phi=0)$ or on the opposite phase $(\phi=\pi)$. This phase ambiguity (equal to $\pi$ ) cannot be resolved inside the synchronizer. Therefore, a phase ambiguity resolution scheme must be included. Fig. 3 proves the reliability of the analytic expression of the S-curve (17) which will allow us to define the carrier phase loop equivalent linear and nonlinear models. Using these models we present in the next section a new nonlinear study for the GMSK carrier phase loop. Obtained results can be generalized for all carrier phase loop employing semi-sinusoidal phase detector $(S(\phi) \propto$ $\sin (2 \phi))$ such as the BPSK Costas loop [7]. 


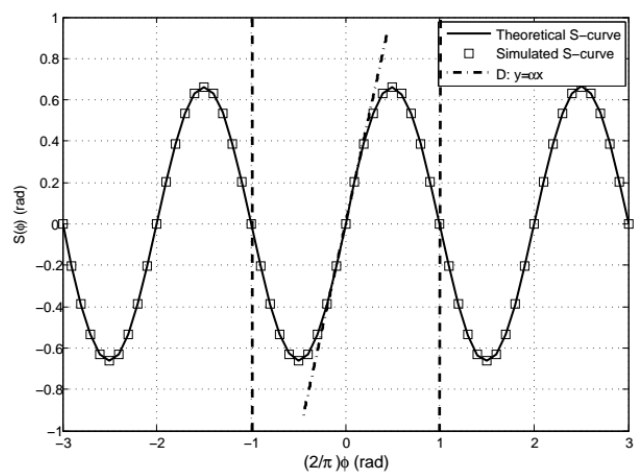

Figure 3. S-curve of the phase detector

\section{CARRIER PHASE LOOP NONLINEAR ANALYSIS}

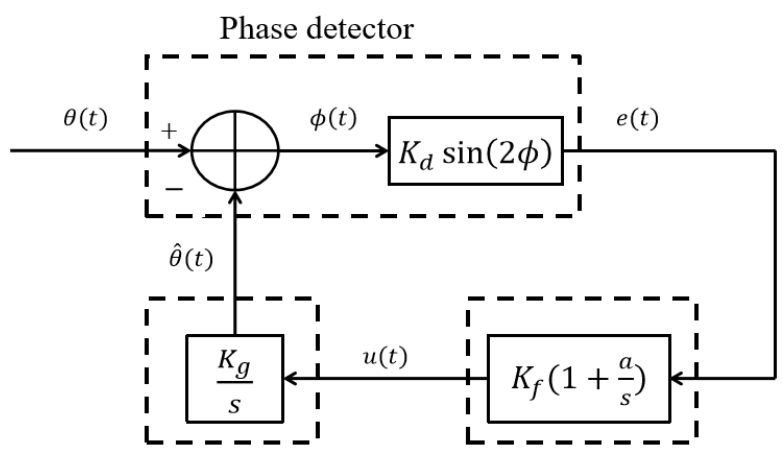

NCO Loop Filter

Figure 4. Nonlinear PLL equivalent model

A block diagram of the carrier phase loop nonlinear equivalent model is shown in Fig. 4. Where, $\theta(t)$ and $\hat{\theta}(t)$ are the instantaneous phase of the carrier signal and that of the NCO signal, respectively, $e(t)$ represents the error signal and $u(t)$ the loop filter output (or the NCO control signal). $K_{g}$ is known as the $\mathrm{NCO}$ again. Note that the nonlinear analysis has been developed in the continuous time domain. However, the transformation (dividing the loop parameters by the sampling period) used for the digital transition does not directly influence the behavior of the system and the obtained results remain applicable.

In the first part of this section we consider the case of a phase ramp input, i.e.

$$
\theta(t)=\Delta w t+\theta_{0}
$$

where $\Delta w=w_{i}-w_{0}$ is the difference between the carrier signal frequency $w_{i}$ and the center frequency of the NCO $w_{0}$ and $\theta_{0}$ is the phase offset at $t=0$.

1) System nonlinear equation: firstly, we derive the nonlinear differential equation governing the system behavior. The mathematical model of the NCO is:

$$
\frac{d \hat{\theta}(t)}{d t}=K_{g} u(t)
$$

The transfer function of the loop filter can be represented in the time domain as

$$
\frac{d u(t)}{d t}=K_{f}\left(a e(t)+\frac{d e(t)}{d t}\right)
$$

By Differentiating (20) with respect to $t$ and substituting (21) into the result, we obtain

$$
\frac{d^{2} \hat{\theta}(t)}{d t^{2}}=K_{g} K_{f}\left(a e(t)+\frac{d e(t)}{d t}\right)
$$

The time derivative of the error signal $e(t)$ can be replaced by

$$
\frac{d e(t)}{d t}=\frac{d e(t)}{d \phi(t)} \frac{d \phi(t)}{d t}
$$

where $\phi(t)=\theta(t)-\hat{\theta}(t)$. In the nonlinear loop model, the phase detector is represented by the analytic expression of the S-curve (17)

$$
e(t)=K_{d} \sin (2 \phi(t))
$$

where $K_{d}=E_{b}^{2}\left(R^{2}(0)-2 R^{2}(1)\right)$.

By using (22), (23) and (24), the system differential equation can be expressed as

$$
\frac{d^{2} \phi(t)}{d t^{2}}+2 G \cos (2 \phi(t)) \frac{d \phi(t)}{d t}+G a \sin (2 \phi(t))=0
$$

where $G=K_{d} K_{f} K_{g}$ is the closed-loop gain.

Finally, we normalize the equation (25) by the closed-loop gain $G$

$$
\frac{d^{2} \phi(t)}{d \tau^{2}}+2 \cos (2 \phi(t)) \frac{d \phi(t)}{d \tau}+a^{\prime} \sin (2 \phi(t))=0
$$

where $\tau=G t$ and $a^{\prime}=a / G$ is the normalized integrator gain.

The system equation (26) is a second-order nonlinear differential equation which does not have a known analytic solution. Therefore, the phase plan is used to analyze the nonlinear system behavior.

2) Equilibrium points: decompose (26) in a set of equations as follows

$$
\begin{gathered}
\frac{d \phi(t)}{d \tau}=\dot{\phi}(t) \\
\frac{d \dot{\phi}(t)}{d \tau}=-2 \cos (2 \phi(t)) \dot{\phi}(t)-a^{\prime} \sin (2 \phi(t))
\end{gathered}
$$

where $(\phi(t), \dot{\phi}(t))$ are the phase and the frequency offset respectively.

By definition, $M\left(\phi_{0}, \dot{\phi}_{0}\right)$ is an equilibrium point for the system described by (26) if it satisfies the following conditions

$$
\frac{d \phi_{0}(t)}{d \tau}=0
$$




$$
\frac{d \dot{\phi}_{0}(t)}{d \tau}=0
$$

Then the equilibrium point is defined as follows

$$
\begin{gathered}
\dot{\phi}_{0}=0 \\
\phi_{0}=k \frac{\pi}{2}, k \in \mathbb{Z}
\end{gathered}
$$

It can be shown [6] that the equilibrium point $M\left(\phi_{0}, \dot{\phi}_{0}\right)$ is a spiral stable node if $k$ is even and an unstable equilibrium point if $k$ is odd.

According to (31) and (32), once the tracking phase is reached, the frequency offset $\dot{\phi}$ will be completely eliminated, but the loop can lock on the opposite phase $(\phi=\theta+\pi)$ since the points $M(\phi=n \pi, \dot{\phi}=0), n=1,3,5 \ldots$, are also stable equilibrium points.

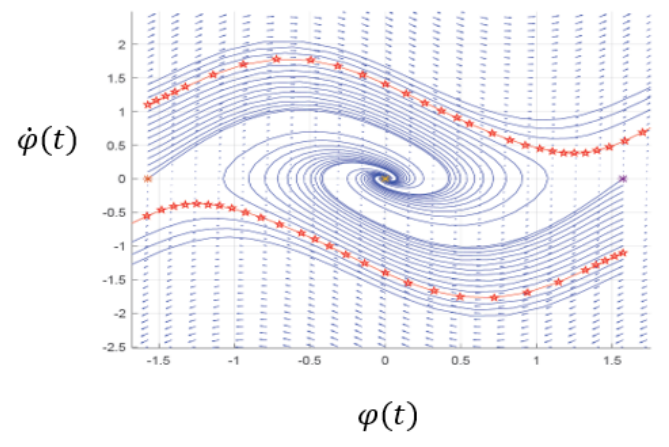

Figure 5. Phase Plane

3) Phase plane: by dividing (28) by (27), we obtain

$$
\frac{d \dot{\phi}}{d \phi}=-2 \cos (2 \phi(t))-\frac{a^{\prime} \sin (2 \phi(t))}{\dot{\phi}(t)}
$$

For an initial condition $M_{0}(\phi(t=0), \dot{\phi}(t=0))$, the equation (33) allows us to calculate (by using a numerical calculator) the tangent at each instant $t_{0}$ and so to obtain point by point the trajectory describing the evolution of the phase and the frequency offset during the time. By moving the initial point $M_{0}$ and using this approach, we can plot the so-called phase plane of the carrier phase loop. Fig.5 illustrates the phase plane of the carrier phase loop around the equilibrium point $M_{0}(\phi=0, \dot{\phi}=0)$ and during the first period $\left(\phi \in\left[-\frac{\pi}{2}, \frac{\pi}{2}\right]\right.$ ) for $a^{\prime}=0.25$ (this value corresponds to the range of the damping factor usually used in practice [6]). For small $|\dot{\phi}|$, the trajectories have a spiral form and approach the local stable equilibrium point without $\phi$ advancing multiples of $\pi$ (this phenomenon is called cycle slipping), at this point the carrier phase loop is in locked state. For very large $|\dot{\phi}|$ the right-hand side of (33) is dominated by the first term $\cos (2 \phi(t))$ and the trajectories in this area are nearly sinusoidal and slip one or more cycle before arriving at an equilibrium point (the carrier loop is in pull-in process). The two particular trajectories (plotted in red on Fig.5) separating these two types of trajectories (spiral and sinusoidal) are known as separatrix.

4) Lock-in range: Assume that the PLL is initially in locked state (in an equilibrium point of the phase plane). The pull-out frequency $\Omega_{p o}$ is defined as the maximum value (in absolute value) of the frequency offset that may arise between the NCO and the carrier signal and still the carrier loop is able to relock without slipping any cycle (return to the same equilibrium point). Exceeding this frequency, the PLL will slip one or more cycle before reaching another stable equilibrium point (relocking). The frequency interval $\left[-\Omega_{p o}, \Omega_{p o}\right]$ is known as the lock-in range in the PLL theory. The pull-in process can be too slow and unreliable for many applications. Therefore, the lock-in range is very important parameters to satisfy the required transmission constraints.

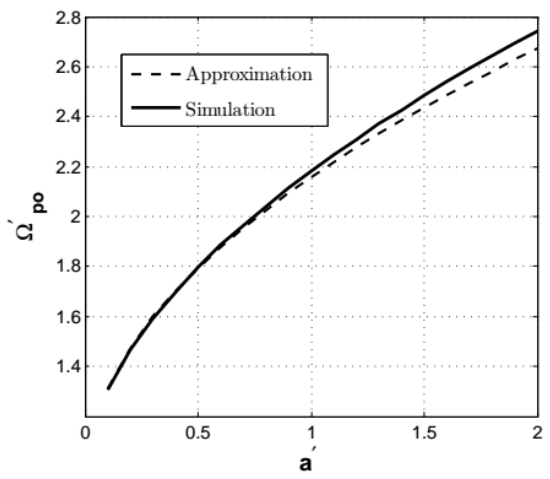

Figure 6. Pull-out frequency

According to the phase plane description above and the lock-in definition, the pull-out frequency can be determined as the intersection of the frequency axis and the separatrix. Fig. 6 illustrates the pull out frequency $\Omega_{p o}$ as a function of the normalized loop filter integrator gain $a^{\prime}$. An approximate analytic expression (34) of the pull-in frequency is also plotted in dashed-line. Using the analytic expression of the phase detector S-curve (17) and the pull-out frequency (34), we are able to well configure the carrier phase loop (the closedloop gain and the integrator gain) and assure the system performance in terms of acquisition time.

$$
\Omega_{p o}^{\prime}=1.24\left(0.74+\sqrt{a^{\prime}}\right)
$$

The left-hand side of Fig.7 illustrates the phase and frequency offset of Fig. 2 in two different scenarios. First, the initial frequency offset is smaller than the pull-out frequency. The carrier loop returns to the locked-state without slipping any cycle. In the second scenarios the initial frequency offset is larger than the pull-out frequency. In this, case the carrier loop slip. one $\pi$-cycle and converges to the stable point $(\phi=\pi, \dot{\phi}=0)$. On the right-hand side, the two corresponding trajectories of the phase plane are plotted and we notice that we obtain exactly the same results which 

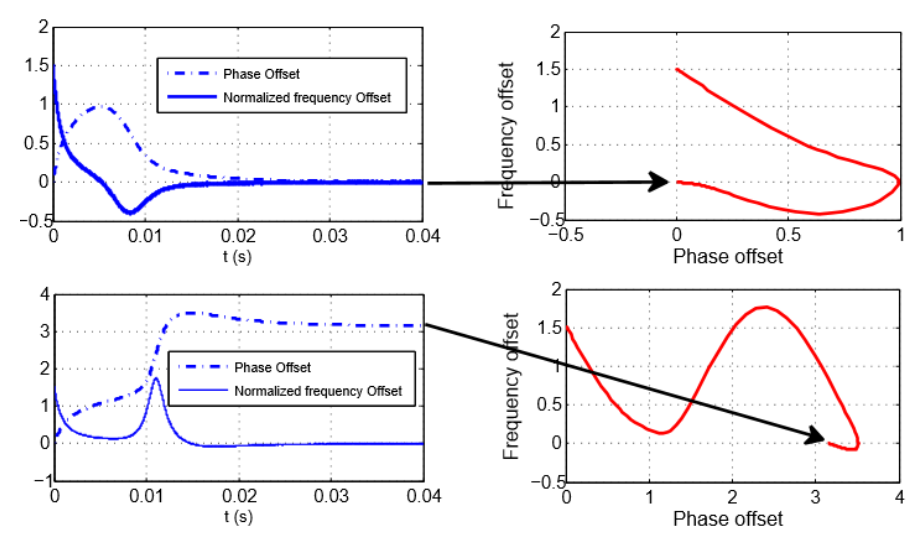

Figure 7. Carrier phase loop nonlinear behavior

proves the reliability of this analysis method.

5) Pull-in time: starting from an initial frequency offset $\dot{\phi}_{0}$ larger than the pull-out frequency, the time required for the carrier phase loop to arrive at an equilibrium stable point (locked state) is known as the pull-in time. In this section, an approximation of this parameter is presented. Firstly, a few assumptions must be made:

1) The frequency offset changes slowly from a cycle to another.

2) The phase detector output contains an "AC" component and a "DC" component $v_{D C}$.

3) The "DC" component has a negligible effect on the NCO frequency.

4) The NCO is controlled by the loop filter integrator output $v_{\text {int }}$ only.

Under these assumptions, the instantaneous frequency offset can be expressed as

$$
\begin{gathered}
\dot{\phi}(t)=\dot{\phi}_{0}-a K_{f} v_{\text {int }}(t) \\
\frac{d \dot{\phi}(t)}{d t}=-a K_{f} v_{D C}(t)
\end{gathered}
$$

It can be shown that

$$
v_{D C}(t)=\frac{K_{d} G}{2 \dot{\phi}(t)}
$$

Substituting (37) in (36), we obtain

$$
d t=-\frac{2}{a G^{2}} \dot{\phi}(t) d \dot{\phi}(t)
$$

Finally, by integrating (38) between $\dot{\phi}_{0}$ and 0 (which presents a frequency locked state), the pull-in time is given by

$$
T_{\text {pull }}=\frac{\dot{\phi}_{0}^{2}}{a G^{2}}
$$

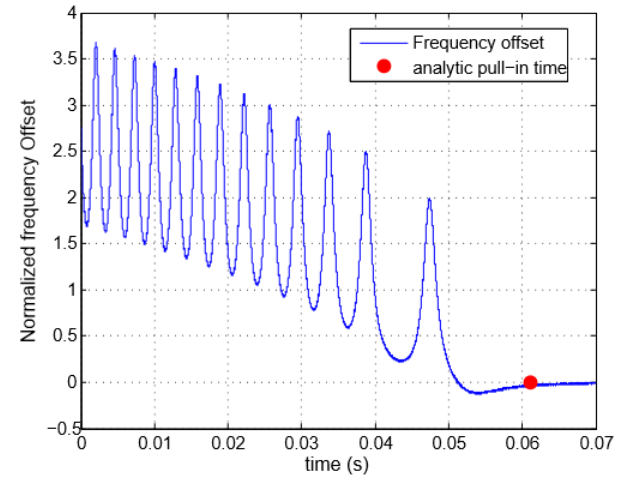

Figure 8. Carrier phase loop frequency offset in pull-in process

Fig.8 illustrates the frequency offset evolution during the time for a closed-loop gain $G=500$, a loop filter integrator gain $a=125$ and an initial frequency offset $\dot{\phi}_{0}=2 \pi \times 220 \mathrm{~Hz}$. For this configuration, the pull-out frequency $\Omega_{p o}=2 \pi \times$ $120 \mathrm{~Hz}$. Using (39), the analytic pull-in time is equal to 0.0611 seconds. The performance of this carrier phase synchronizer in terms of phase error variance and bit error rate can be found in [8].

\section{CONCLUSION}

In this paper, a nonlinear analysis for a GMSK carrier phase recovery scheme was presented. Using, the derived analytic expression of the phase detector S-curve, the nonlinear carrier phase loop equivalent model was defined and investigated by the phase plane method. Several computer simulation were carried out in different scenarios and the reliability of the obtained resulted was proved. This study can be generalized for all carrier phase loop with a semi-sinusoidal phase detector $(S(\phi) \propto \sin (2 \phi))$ which presents the important contribution of our paper.

\section{REFERENCES}

[1] CCSDS Recommendations For Space Data System Standards. Radio Frequency and Modulation Systems, Part 1, Earth Stations and Spacecraft. CCSDS 401.0-B Blue Book, June 2001.

[2] P. Laurent, "Exact and approximate construction of digital phase modulations by superposition of amplitude modulated pulses (amp)," IEEE Transactions on Communications, vol. 34, no. 2, pp. 150-160, Feb 1986.

[3] M. K. Simon, Bandwidth-efficient digital modulation with application to deep space communication. Wiley and Sons, 2003.

[4] E. Vassallo and M. Visintin, "Carrier phase synchronization for gmsk signals," International Journal of Satellite Communication, pp. 391-415, 2002.

[5] S. Shambayati and D. K. Lee, "Gmsk modulation for deep space applications," pp. 1-13, 2012.

[6] J. L. Stensby, Phase-Locked LOOPS Theory and Applications. CRC Press, 1997.

[7] U. Mengali and A. N. D’Andrea, Synchronisation Techniques for Digital Receivers. PLENUM Press, 1997.

[8] M. A. Jhaidri, A. Thomas, and C. Laot, "Performance analysis of gmsk carrier synchronization loop phase detector at very low snr," Europeen space agency TTC Workshop, Noordwijk Netherlands, 2016. 